\title{
O ENSINO/APRENDIZAGEM DE MÉTODOS E TÉCNICAS DE INVESTIGAÇÃO SOCIAL \\ Contributos para o desenvolvimento de uma cultura pedagógica
}

\author{
TEACHING/LEARNING SOCIAL RESEARCH METHODS \\ AND TECHNIQUES \\ A contribution for developing a pedagogical culture
}

\author{
José de São José \\ Centro Interdisciplinar de Ciências Sociais (CICS.NOVA) \& Universidade do Algarve, Faculdade de Economia. \\ Campus de Gambelas, Edifício 9, 8005-139 Faro, Portugal. Email: jsjose@ualg.pt
}

\begin{abstract}
Resumo: O ensino/aprendizagem de métodos e técnicas de investigação no domínio das ciências sociais expandiu-se significativamente ao longo das últimas décadas, prevendo-se que continue a expandir-se e a adquirir uma relevância ainda maior devido à crescente valorização da capacidade de investigação por parte de universidades, centros de investigação, empresas e outras organizações. Contudo, não existe uma cultura pedagógica no ensino/aprendizagem de métodos e técnicas de investigação social, isto é, não temos uma discussão nem investigação sistemáticas sobre todos os aspetos que dizem respeito ao ensino/aprendizagem destes métodos e técnicas. Através da partilha da sua experiência de lecionação de unidades curriculares desta área científica, e da reflexão que realiza sobre esta experiência à luz da literatura especializada, o autor deste artigo procura contribuir para a discussão dos aspetos pedagógicos no ensino/aprendizagem de métodos e técnicas de investigação social, discussão importante, mas que tarda em se iniciar de uma forma definitiva em Portugal. Após concluir que as suas práticas pedagógicas vão ao encontro do preconizado pela literatura revista, o autor deste artigo vai para além desta literatura, propondo princípios pedagógicos (e práticas pedagógicas) que permitem ultrapassar algumas das suas principais limitações.
\end{abstract}

Palavras-chave: cultura pedagógica, ensino/aprendizagem, métodos e técnicas de investigação, ciências sociais.

\begin{abstract}
The teaching and learning of social research methods has expanded significantly over the last decades and is expected to continue to expand and become even more relevant due to the increasing value that universities, research centers, companies and other organizations ascribe to research capacity. However, there is no pedagogical culture in teaching/learning social research methods, i.e., we do not have a systematic discussion or research on all aspects of teaching/learning these methods. By sharing his experience in teaching curricular units of this scientific area, and reflecting on this experience in light of the specialized literature, the author contributes to the discussion of pedagogical aspects in teaching/learning social research methods, an important and overdue discussion in Portugal. After concluding that his pedagogical principles and practices are in line with those recommended by the revised literature, the author proposes pedagogical principles (and pedagogical practices) that allow to overcome some of its main limitations.
\end{abstract}

Keywords: pedagogical culture, teaching/learning, social research methods, social sciences. 


\section{Introdução}

Os métodos e técnicas de investigação social (MTIS) - devendo-se entender a expressão investigação social como sinónimo de investigação no domínio das ciências sociais - têm a sua origem na época moderna (séc. XVIII), com o advento da industrialização, da urbanização e do capitalismo. Nessa altura, as entidades públicas começaram a sentir a necessidade de compilar informação de diversa natureza sobre as populações, tendo-se então criado alguns instrumentos e procedimentos de recolha e análise de dados. Não obstante os vários desenvolvimentos então ocorridos, sobretudo no domínio da estatística, a institucionalização dos MTIS enquanto campo académico só se dá definitivamente no final do séc. XIX, início do séc. XX, por intermédio do surgimento das primeiras obras e das primeiras disciplinas universitárias sobre o tema (Alastalo, 2008; Crothers \& Platt, 2010). O livro da autoria de Émile Durkheim intitulado Les règles de la méthode sociologique, publicado pela primeira vez em 1895, é considerado por muitos autores o primeiro manual de MTIS (Alastalo, 2008). Este manual foca-se na investigação quantitativa, surgindo bastantes décadas mais tarde, mais precisamente em 1967, aquele que é considerado o primeiro manual de investigação qualitativa, intitulado The Discovery of Grounded Theory, da autoria de Barney Glaser e Anselm Strauss (Alastalo, 2008). O surgimento mais tardio deste último manual justificar-se-á pela afirmação, também mais tardia, da investigação qualitativa na academia. Nos Estados Unidos da América, a Universidade de Chicago e a Universidade de Columbia iniciaram o ensino de MTIS nas primeiras décadas do séc. XX.

Desde o início do séc. XX que o desenvolvimento, ensino/aprendizagem e utilização dos MTIS se tem vindo a expandir de uma forma muito significativa no campo académico (Kilburn, Nind \& Wiles, 2014; Nind, Kilburn \& Luff, 2015). Por exemplo, nas universidades, o ensino de MTIS está hoje disseminado por cursos de diferentes áreas científicas e de diferentes ciclos de estudo (Kilburn et al., 2014; Nind et al., 2015) e o volume de manuais e de outras publicações sobre a temática é deveras impressionante (Alastalo, 2008), existindo mesmo revistas científicas especializadas sobre metodologias no domínio das ciências sociais, como por exemplo o International Journal of Social Research Methodology e o Forum: Qualitative Social Research.

Todavia, não é apenas no mundo académico que constatamos a importância dos MTIS. Também as empresas apostam cada vez mais na recolha e tratamento de informação relevante para a sua atividade (Nind et al., 2015). No fundo, as universidades, os centros de investigação e as empresas estão cada vez mais preocupadas com a capacidade de investigação, muito particularmente com a capacidade de recolha 
e tratamento de informação, sendo esta entendida como fundamental para competir nas sociedades contemporâneas em que a informação tem um papel vital (Kilburn et al., 2014; Nind et al., 2015).

O desenvolvimento da capacidade de investigação está, naturalmente, dependente da formação em MTIS. O enfoque na formação e no ensino de MTIS é hoje muito visível em países como os Estados Unidos da América, mas também na União Europeia (Nind et al., 2015). Contudo, vários autores têm chamado a atenção para o facto de que são raros os professores e formadores que têm formação suficiente sobre como ensinar MTIS (Earley, 2014). A inexistência ou insuficiência desta formação tem diversas causas: i) a literatura disponível sobre a pedagogia dos MTIS ainda é escassa (Wagner, Garner \& Kawulich, 2011; Lewthwaite \& Nind, 2016), devendo-se entender por pedagogia não apenas as práticas dos professores em contextos de ensino/aprendizagem, mas também as suas perceções sobre o que é significativo, importante e relevante no processo de ensino/aprendizagem (Nind et al., 2015); ii) para além de escassa, esta literatura é limitada, pois está confinada a determinados métodos/áreas disciplinares (Wagner et al., 2011), não aborda os modos como os estudantes aprendem e o que realmente aprendem (Earley, 2014), não discute os métodos de avaliação dos estudantes (Earley, 2014), e não se baseia, na sua maior parte, em investigação empírica (Wagner et al., 2011); iii) e os professores continuam a negligenciar as questões pedagógicas na sua prática letiva (Wagner et al., 2011), embora esta realidade esteja a mudar (Kilburn et al., 2014).

Nesta ordem de ideias, temos, num dos lados da balança, uma sólida institucionalização e utilização dos MTIS em diversas esferas sociais, aliada a uma crescente importância atribuída à capacidade de investigação e, concomitantemente, à capacidade de ensinar/formar na área dos MTIS e, no outro lado da balança, a inexistência de uma cultura pedagógica no domínio dos MTIS (Wagner et al., 2011, p. 75). Na ótica de Wagner et al. (2011, p.75), cultura pedagógica refere-se ao seguinte: "troca de ideias num clima de debate sistemático, investigação e avaliação incluindo todos os aspetos do ensino e aprendizagem numa dada matéria". A inexistência de uma cultura pedagógica no domínio dos MTIS comporta várias consequências: “Deixados por sua própria conta, os professores de métodos de investigação contam com uma rede de pares, literatura dispersa e muitas tentativas e erros à medida que desenvolvem e aprimoram os seus próprios cursos de métodos de investigação" (Earley, 2014, p. 243).

Esta ausência de cultura pedagógica torna-se ainda mais problemática dadas as representações e as atitudes negativas da generalidade dos estudantes face às unidades curriculares (UC) de MTIS. A evidência empírica mostra que os estudantes: i) acham que estas UC são aborrecidas e irrelevantes para as suas necessidades 
(Howard \& Brady, 2015); ii) temem os conteúdos, especialmente das UC de métodos quantitativos, pois associam-nos à matemática (Kilburn et al., 2014); iii) e julgam que não é necessário obter uma boa classificação em UC de MTIS para obter sucesso na licenciatura (Kilburn et al., 2014). Howard e Brady (2015) acrescentam que, nos contextos académicos dominados pela viragem crítica (critical turn), que se traduz, entre outras coisas, numa crítica pós-moderna e pós-estruturalista aos métodos convencionais de investigação, o desinteresse dos estudantes é ainda mais acentuado como resultado do ceticismo que esta viragem crítica provoca relativamente às mais-valias de aprender MTIS.

Com o propósito de contribuir para o desenvolvimento de uma cultura pedagógica no ensino/aprendizagem dos MTIS, este artigo partilha, com a comunidade académica, a experiência do autor no ensino de uma UC da área dos métodos qualitativos de um curso de licenciatura em Sociologia, bem como a sua reflexão sobre esta experiência, tendo como pano de fundo a literatura relevante revista. $\mathrm{O}$ autor deste artigo propõe princípios pedagógicos (e práticas pedagógicas) que permitem ultrapassar algumas das principais limitações desta literatura.

\section{Ensinar métodos e técnicas de investigação social: $O$ que nos diz a literatura?}

Como foi referido anteriormente, a literatura sobre a pedagogia dos MTIS ainda é escassa e limitada. Não obstante, três publicações - uma revisão da literatura (Kilburn et al., 2014) e dois estudos empíricos (Lewthwaite \& Nind, 2016; Nind, 2019) - fornecem informação relevante sobre esta temática, embora existam outros estudos menos abrangentes, como por exemplo o realizado por Howard e Brady (2015), que propõe uma pedagogia construtivista.

A revisão da literatura (Kilburn et al., 2014) inclui 24 publicações que dão conta das abordagens pedagógicas adotadas por professores de MTIS (de natureza quantitativa e qualitativa), que exerciam a sua atividade docente em diferentes ciclos de estudo (licenciatura e pós-graduações) no domínio das ciências sociais (sobretudo no Norte da América). Esta revisão constatou que as diversas abordagens pedagógicas refletiam preocupações com três objetivos pedagógicos complementares e inter-relacionados: $i$ ) tornar a investigação visível (making the research visible); ii) aprender através da realização de atividades de investigação (learning by doing research); iii) e refletir sobre o processo de investigação (reflection on the research process). O primeiro objetivo pedagógico procura colocar os estudantes em contacto com atividades de investigação, quer através da discussão de exemplos ilustrativos, quer através da realização de determinadas atividades por parte dos 
estudantes (ex.: avaliação crítica de resultados de investigação e visionamento de filmes/documentários relevantes). A aprendizagem ativa, que na ótica de Bonwell e Eison (1991, p. 2) "implica os estudantes a fazerem coisas e a pensarem nas coisas que estão a fazer" é considerada pela generalidade das publicações revistas como um paradigma pedagógico privilegiado para tornar a investigação visível. O segundo objetivo pedagógico está estritamente relacionado com o primeiro, e inclui ora a realização de atividades de investigação em contextos reais, ora a realização de atividades com dados empíricos reais (ex.: envolver os estudantes na realização e transcrição de entrevistas, e na criação de bases de dados em softwares de investigação quantitativa, etc.). O terceiro objetivo pedagógico implica estimular os estudantes para refletirem sobre o que fazem, isto é, sobre as atividades que realizam e sobre as aprendizagens obtidas (ex.: refletir por que é que as técnicas de investigação se devem usar de determinada maneira, fazer um balanço sobre as aprendizagens consolidadas e as aprendizagens ainda não consolidadas).

O estudo realizado por Lewthwaite e Nind (2016), através de um painel de 21 especialistas/professores de MTIS oriundos de diversos continentes (Europa, América, África e Ásia), com experiência ao nível do ensino pós-graduado no domínio das ciências sociais, chega a resultados similares. Este estudo apenas acrescenta ou reforça algumas abordagens, tais como a aprendizagem baseada no problema (problem-based learning), em que, por exemplo, um problema real de investigação é usado como ponto de partida para abordar determinadas questões metodológicas.

Por último, o estudo realizado por Nind (2019), também realizado junto de um conjunto de especialistas/ professores de MTIS (de âmbito internacional), que participavam em programas de doutoramento e/ou em programas de formação avançada para investigadores no domínio das ciências sociais, procurou saber como é que estes ensinavam os conteúdos programáticos aos seus estudantes/formandos. Este estudo partiu do conceito de conhecimento pedagógico do conteúdo (pedagogical content knowledge) (PCK), muito usado no domínio da formação de professores, que remete para " (...) o conhecimento que os professores desenvolvem ao longo do tempo, através da sua experiência, sobre como ensinar um determinado conteúdo de um modo particular a fim de levar a uma melhor compreensão do aluno" (Loughran, Berry \& Mulhall, 2012, p. 7). Este conceito advoga que o ensino envolve algo mais do que a simples transmissão de conhecimento aos estudantes, e que a aprendizagem dos estudantes envolve algo mais do que a simples absorção de informação para posterior regurgitação (Loughran et al., 2012, p. 7).

Nind (2019) constata que existe um PCK genérico, um PCK específico para os MTIS, e ainda PCK específicos para os métodos quantitativos e para os métodos qualitativos. No que toca ao PCK genérico, os resultados apontam para a relevância de 
um conjunto de estratégias pedagógicas relacionadas com os seguintes aspetos: i) organização (organização dos estudantes e dos conteúdos programáticos); ii) ritmo das atividades a desenvolver nas aulas; iii) abordagens pedagógicas gerais (aprendizagem experiencial, aprendizagem ativa, aprendizagem centrada no estudante); iv) diversidade dos estudantes; $v$ ) necessidades dos estudantes; vi) atividades/técnicas para tornar os conteúdos relevantes e cativantes.

Por sua vez, no respeitante ao PCK específico para os MTIS, Nind (2019) isola as seguintes estratégias pedagógicas: i) ensinar com, através e sobre dados - dados usados como um conector pedagógico (pedagogic hook); ii) tomar a decisão pedagógica sobre que dados utilizar (dados produzidos pelos próprios estudantes ou dados secundários); iii) tomar a decisão pedagógica sobre o tipo de envolvimento dos estudantes com os dados (aprendizagem experiencial, isto é, aprendizagem com dados recolhidos pelos próprios estudantes, ou aprendizagem ativa ou baseada no problema, em que os estudantes trabalham ativamente com dados não recolhidos pelos próprios); iv) narração (traçar a história de uma pesquisa empírica com dados reais e credíveis); $v$ ) identificar a teoria por detrás da lógica, a lógica por detrás das decisões, e as decisões por trás da ação (teoria e racionalidade subjacentes ao processo de investigação).

Relativamente ao PCK específico para os métodos quantitativos, as estratégias pedagógicas identificadas são as seguintes: i) traduzir a linguagem técnica, em linguagem não técnica/linguagem visual; ii) organizar um processo linear de aprendizagem (estruturar uma sequência de conteúdos e atividades com um grau crescente de dificuldade); iii) desenvolver materiais para o livro de atividades que apoia o processo de ensino/aprendizagem.

Por último, no que diz respeito ao PCK específico para os métodos qualitativos, as estratégias pedagógicas encontradas por Nind (2019) são as seguintes: $i$ ) explicitar os pontos de vista de partida (standpoints), quer de natureza epistemológica, quer de natureza teórica; ii) desenvolver o espírito crítico e a reflexividade junto dos estudantes; iii) incorporar (embodying) os métodos/dados (ex.: envolver profundamente os estudantes nos dados, ora através da leitura em voz alta de excertos de entrevistas, ora através da escrita das suas próprias histórias de vida ou da suas auto-etnografias).

Enquanto as duas primeiras publicações dão ênfase aos objetivos pedagógicos, a terceira foca-se no modo como os MTIS são efetivamente ensinados. Por outras palavras, enquanto as duas primeiras publicações se direcionaram para o quêe (objetivos pedagógicos), a última preocupa-se em dar conta do como (estratégias e atividades pedagógicas usadas).

Conforme mencionado na introdução, a literatura sobre a pedagogia dos MTIS ainda é escassa e exibe diversas limitações. As limitações que mais sobressaem nas 
três publicações acabadas de rever são: i) a ausência de discussão dos métodos de avaliação dos estudantes; ii) a ausência de discussão do papel da avaliação no processo de ensino/aprendizagem; iii) a negligência das perspetivas dos estudantes sobre a pedagogia dos MTIS.

O modo como se ensina/aprende não se dissocia do modo como se avalia. Por outras palavras, ensino/aprendizagem e a avaliação são duas faces da mesma moeda. Daí a ausência de discussão da questão da avaliação, não só do seu modus operandi, mas também do seu papel no próprio processo de ensino/aprendizagem, ser particularmente problemática. $\mathrm{O}$ autor deste artigo procurou ultrapassar estas limitações no âmbito da lecionação de uma UC de MTIS, nos termos que se descrevem na próxima seção.

A negligência das perspetivas dos estudantes sobre a pedagogia dos MTIS também é problemática, dado que a prática de ensinar (perspetivas dos docentes) não se dissocia da prática de aprender (perspetivas dos estudantes). As práticas de ensino/aprendizagem estabelecem-se no quadro de uma relação entre professores e estudantes. Voltar-se-á a esta limitação na conclusão.

A estas três limitações pode-se ainda acrescentar o foco predominante nos cursos de pós-graduação, o que poderá limitar o alcance e a pertinência desta literatura.

\section{Ensinar métodos e técnicas de investigação social: partilha de uma experiência}

Nesta seção descreve-se uma experiência de ensino de MTIS que se iniciou no ano letivo 2007-2008. Na altura, a UC lecionada tinha a designação de Métodos e Técnicas de Investigação Sociológica I (focada sobre a investigação qualitativa), tendo sido alterada no ano letivo 2016-2017 (no seguimento de uma mudança na estrutura curricular e no plano de estudos da licenciatura) para Métodos e Técnicas de Investigação Qualitativa (MTIQual). Esta UC é parte integrante do plano de estudos da licenciatura em Sociologia (em uma Universidade portuguesa, $2^{\circ}$ ano, $2^{\circ}$ semestre) e tem os seguintes objetivos de aprendizagem: $i$ ) identificar e explicar as principais características da investigação qualitativa e as suas principais etapas; ii) entender a multiplicidade de abordagens no domínio da investigação qualitativa; iii) definir perguntas de partida e realizar revisões da literatura; iv) desenvolver modelos de análise no âmbito de investigações qualitativas; $v$ ) compreender as principais técnicas de amostragem não probabilística; vi) delinear planos de amostragem; vii) identificar e explicar as principais técnicas de recolha de dados qualitativos; viii) conceber guiões de entrevista semiestruturados; $i x$ ) explicar as principais técnicas de 
condução de entrevistas qualitativas; $x$ ) descrever os principais critérios de avaliação da qualidade da investigação qualitativa; xi) desenvolver o pensamento crítico, as competências de comunicação oral e escrita, e a capacidade de trabalho em equipa. Os objetivos de aprendizagem (assim como os conteúdos programáticos) foram definidos não só através da análise de Fichas de Unidade Curricular (FUC) idênticas pertencentes a outros cursos da área das ciências sociais, tanto de universidades nacionais como estrangeiras, como também através da partilha de experiências e da troca de impressões com colegas que lecionam UC de MTIS. É de referir que a FUC de MTIQual foi oportunamente aprovada pelo Conselho Pedagógico da Faculdade em que a licenciatura se integra, assim como pela Agência de Avaliação e Acreditação do Ensino Superior.

\section{Princípios pedagógicos e estratégia pedagógica geral}

Uma vez definidos os objetivos de aprendizagem e os conteúdos programáticos, surgiu a necessidade de pensar nos métodos de ensino/aprendizagem e nos métodos de avaliação. Apesar de as FUC então analisadas de cursos de outras universidades terem informação suficiente sobre os métodos de avaliação, a informação sobre os métodos de ensino/aprendizagem era escassa e incompleta. A isto adicionou-se também a escassa e incompleta literatura sobre a pedagogia dos MTIS. Perante este cenário, o docente recorreu a um conjunto de experiências e saberes para definir um determinado número de princípios pedagógicos para a sua docência, nomeadamente o modo como tinha sido ensinado pelos seus professores, a sua experiência de investigação, bem como alguma informação geral sobre pedagogia no ensino superior. No que respeita a este último aspeto, o docente aderiu ao paradigma da aprendizagem ativa, tal como foi explanado por Bonwell e Eison (1991), paradigma determinante na estruturação dos três grandes princípios seguidos no ensino da UC de MTIQual, principalmente do primeiro princípio (o termo paradigma é aqui usado como conjunto de pressupostos e de métodos, distinguindo-se de estratégia, aqui entendida como sinónimo de plano de ação).

O primeiro princípio pedagógico seguido na UC de MTIQual estabelece que o ensino/aprendizagem de MTIS é mais eficaz se envolver a participação ativa dos estudantes na realização de atividades práticas (em vez de lhes atribuir um papel passivo como meros recetáculos dos conhecimentos transmitidos pelo docente). Segundo, o princípio de que a avaliação, mais concretamente a avaliação contínua, pode (e deve) ser uma componente importante da estratégia de ensino/aprendizagem, interligando-se com as atividades letivas de natureza teórico-prática. A avaliação não deve ter a função exclusiva de avaliar, mas também de contribuir para o reforço do ensino/aprendizagem, principalmente quando o estudante tem 
a oportunidade de receber comentários e sugestões do docente sobre o trabalho que é sujeito a avaliação. Terceiro, o princípio de que a avaliação de competências e conhecimentos deve ser precedida pelo treino/aplicação prática dessas mesmas competências e conhecimentos. A concretização deste princípio contribui para aumentar a confiança dos estudantes e para diminuir a ansiedade relacionada com a avaliação. O segundo e o terceiro princípios ultrapassam duas das limitações da literatura revista sobre a pedagogia dos MTIS, nomeadamente, a ausência de discussão dos métodos de avaliação dos estudantes e do papel da avaliação no processo de ensino/aprendizagem.

Com base nestes princípios, e considerando a dimensão máxima da turma (30 alunos), o docente tomou as seguintes decisões. As aulas são de natureza teórico-prática, significando uma constante mobilização, de forma articulada, da teoria e da prática ao longo do semestre. As aulas teórico-práticas interligam-se com a avaliação contínua, interligação esta que se inicia após a realização das aulas introdutórias, nas quais são discutidos, através de uma abordagem pedagógica tendencialmente mais expositiva, conteúdos básicos de natureza epistemológica e ontológica (correspondentes aos dois primeiros objetivos de aprendizagem). Os estudantes iniciam a avaliação contínua logo após as primeiras aulas do semestre. A forte interligação entre as aulas teórico-práticas e a avaliação contínua é a característica central da estratégia pedagógica (plano de ação) adotada pelo docente.

A avaliação contínua consiste na realização de um portefólio de grupo (trabalho realizado em grupos de 3 a 5 estudantes nos períodos fora das aulas), que é composto por quatro atividades: i) pergunta de partida e revisão da literatura; ii) modelo de análise e perguntas de investigação; iii) plano de amostragem; iv) guião de entrevista semiestruturada. A temática do portefólio de grupo é identificada durante as primeiras aulas através de um processo de negociação entre o docente e os estudantes e, uma vez selecionada, fica a mesma para todos os grupos de trabalho. Seguidamente, o docente seleciona um conjunto reduzido de textos (cerca de 6) sobre a temática (normalmente atas dos congressos da Associação Portuguesa de Sociologia) e torna-os acessíveis aos estudantes através da tutoria eletrónica. Apesar da temática do portefólio de grupo ser a mesma para todos os grupos de trabalho, $\mathrm{o}$ docente assegura que as perguntas de partida e as perguntas de investigação sejam diferentes, garantindo assim que os portefólios sejam também eles diferentes.

A operacionalização da interligação das aulas teórico-práticas com a avaliação contínua processa-se do seguinte modo: i) o docente aborda a questão da pergunta de partida e da revisão da literatura do ponto de vista conceptual e técnico, fornecendo exemplos de perguntas de partida formuladas corretamente e analisando uma revisão da literatura levada a cabo numa investigação qualitativa; ii) seguidamente os 
estudantes realizam atividades práticas (exercícios) relacionadas com estes conteúdos programáticos, nomeadamente definem perguntas de partida sobre um determinado tema e realizam uma mini revisão da literatura com base num número reduzido de textos fornecidos pelo docente; iii) após a finalização destas atividades práticas, os estudantes iniciam a realização da primeira atividade do portefólio de grupo (formulação de uma pergunta de partida sobre a temática previamente selecionada e a realização de uma revisão da literatura com base nos textos também previamente selecionados) e acordam com o docente a data limite para a sua entrega; iv) após a receção de uma versão provisória da primeira atividade realizada por cada um dos grupos de trabalho, o docente, logo que possível, fornece comentários/sugestões sobre cada uma das atividades (estes comentários/sugestões são realizadas, sobretudo, nas tutorias presenciais com a presença de todos os estudantes, permitindo-se desta forma que todos ouçam os comentários sobre as atividades realizadas por todos os grupos de trabalho; $v$ ) os estudantes têm a oportunidade de integrar estes comentários/sugestões nas respetivas atividades antes de procederem à entrega definitiva do portefólio no final do semestre. Estes procedimentos vão-se repetindo até que as quatro atividades sejam entregues ao docente por todos os grupos de trabalho. Por exemplo, no que toca à última atividade, depois de o docente abordar os aspetos concetuais e técnicos sobre as entrevistas semiestruturadas, de discutir um exemplo de entrevista semiestruturada e de solicitar aos estudantes a realização de um mini guião de entrevista semiestruturada, dá-se o início à realização do guião de entrevista semiestruturada que vai ser sujeito a avaliação (este guião é desenvolvido a partir do modelo de análise e das perguntas de investigação definidas na atividade anterior do portefólio de grupo). De igual modo, o docente formula comentários e sugestões sobre esta última atividade, que podem ser integrados na versão final. Importa sublinhar que este modo de interligação entre as aulas e a avaliação contínua foi sendo aperfeiçoado ao longo do tempo através de sucessivas avaliações informais levadas a cabo conjuntamente pelo docente e pelos estudantes.

\section{Confrontação da estratégia pedagógica adotada pelo docente com a literatura anteriormente revista}

Ao longo da sua trajetória de ensino de MTIS, o docente nunca teve formação pedagógica geral nem formação pedagógica específica sobre a pedagogia dos MTIS. Também não recorreu, tal como seria desejável, à leitura de literatura sobre a pedagogia dos MTIS, até porque esta era praticamente inexistente. Isto quer dizer que só muito recentemente iniciou, de uma forma sistemática, a leitura de literatura especializada e tomou conhecimento das publicações anteriormente revistas. Não obstante, os objetivos pedagógicos (Kilburn et al., 2014; Lewthwaite \& Nind, 2016) 
e as estratégias/atividades pedagógicas (Nind, 2019) revistas mais acima encontram-se no modo como as aulas de MTIQual funcionam. Por exemplo, o objetivo pedagógico tornar a investigação visível é alcançado através da discussão de exemplos de componentes do processo de investigação, tais como exemplos de perguntas de partida e de revisões da literatura. Por seu lado, o objetivo pedagógico aprender através da realização de atividades de investigação é perseguido através da realização de atividades práticas, tais como definição de perguntas de partida e realização de uma mini revisão da literatura, assim como através da realização das quatro atividades do portefólio de grupo. Por último, o objetivo pedagógico refletir sobre o processo de investigação atinge-se através do fornecimento de comentários e sugestões sobre as atividades realizadas pelos estudantes, inclusive sobre as atividades que compõem o portefólio de grupo, ações que despoletam nos estudantes a reflexividade e a autocrítica. Na tabela 1 distribuem-se todas as atividades desenvolvidas no âmbito da UC MTIQual pelos três objetivos pedagógicos enunciados por Kilburn e colaboradores (2014) e Lewthwaite e Nind (2016).

Tabela 1 Distribuição das atividades realizadas no âmbito da UC MTIQual pelos três objetivos pedagógicos identificados por Kilburn e colaboradores (2014) e Lewthwaite e Nind (2016)

\begin{tabular}{|c|c|c|}
\hline Tornar a investigação visível & $\begin{array}{l}\text { Aprender através da realização } \\
\text { de atividades de investigação }\end{array}$ & $\begin{array}{l}\text { Refletir sobre o processo } \\
\text { de investigação }\end{array}$ \\
\hline $\begin{array}{l}\text { - Discussão de exemplos } \\
\text { de perguntas de partida } \\
\text { - Discussão de revisões } \\
\text { da literatura } \\
\text { - Discussão de exemplos } \\
\text { de modelos de análise } \\
\text { - Discussão de exemplos } \\
\text { de perguntas de investigação } \\
\text { - Narração de um processo } \\
\text { de conceção de um modelo } \\
\text { de análise e de definição } \\
\text { de perguntas de investigação } \\
\text { - Discussão de exemplos de } \\
\text { planos de amostragem } \\
\text { - Discussão de exemplos de } \\
\text { guiões de entrevista } \\
\text { semiestruturados }\end{array}$ & $\begin{array}{l}\text { - Exercício 1: Definição de perguntas de } \\
\text { partida sobre um tema de investigação } \\
\text { - Exercício 2: Realização de uma mini } \\
\text { revisão da literatura com base num conjunto } \\
\text { limitado de textos } \\
\text { - Realização da primeira atividade do } \\
\text { portefólio de grupo } \\
\text { - Exercício 3: Conceção de um modelo de } \\
\text { análise a partir de uma pergunta de partida e } \\
\text { de uma revisão da literatura } \\
\text { - Exercício 4: Definição de perguntas de } \\
\text { investigação com base no modelo de análise } \\
\text { desenvolvido } \\
\text { - Realização da segunda atividade do } \\
\text { portefólio de grupo } \\
\text { - Exercício 5: Definição de planos de } \\
\text { mostragem com base em populações-alvo e } \\
\text { perguntas de investigação fornecidas pelo } \\
\text { docente } \\
\text { - Realização da terceira atividade do } \\
\text { portefólio de grupo } \\
\text { - Exercício 6: Realização de um mini guião } \\
\text { de entrevista semiestruturada com base num } \\
\text { modelo de análise e de perguntas de } \\
\text { investigação fornecido pelo docente } \\
\text { - Realização da quarta atividade } \\
\text { do portefólio de grupo }\end{array}$ & $\begin{array}{l}\text { - Comentários e sugestões } \\
\text { do docente sobre as atividades } \\
\text { práticas realizadas pelos } \\
\text { estudantes ao longo do } \\
\text { semestre, inclusive sobre cada } \\
\text { uma das atividades do portefólio } \\
\text { de grupo }\end{array}$ \\
\hline
\end{tabular}

Nota: apesar de cada uma das atividades descritas estar inserida apenas num dos objetivos pedagógicos, admite-se que uma certa atividade possa contribuir para a concretização de dois ou três objetivos pedagógicos. 
Relativamente às estratégias pedagógicas identificadas por Nind (2019), importa referir que a aprendizagem ativa, que faz parte do PCK genérico e do PCK relativo aos MTIS é o paradigma adotado pelo docente nas suas aulas (incluindo na concretização da modalidade de avaliação contínua), e que a narração, estratégia integrante do PCK dos MTIS, é praticada pelo docente quando discute exemplos de componentes do processo de investigação, tal como a revisão da literatura, e quando descreve o processo de conceção de um modelo de análise e de definição de perguntas de investigação. A teoria e a racionalidade que subjazem ao processo de investigação, que também integram o PCK dos MTIS, são aspetos que o docente procura constantemente abordar ao longo das aulas e dos comentários que faz sobre as atividades do portefólio de grupo entregues pelos estudantes. No que concerne às estratégias que integram o PCK dos métodos qualitativos, a explicitação dos pontos de vista epistemológicos e ontológicos é realizada nas aulas iniciais, enquanto o desenvolvimento do espírito crítico e da reflexividade junto dos estudantes é algo que o docente persegue em todas as aulas e, muito particularmente, nos momentos em que comenta as atividades do portefólio de grupo. As únicas estratégias que não são usadas pelo docente são ensinar com, através e sobre dados e incorporar os métodos/dados. Isto prende-se com o facto de os objetivos de aprendizagem da UC de MTIQual não prever a utilização de dados de investigação (existe outra UC na licenciatura que se foca na análise qualitativa de dados).

Assim, embora a literatura revista se foque, sobretudo, em cursos de pós-graduação, por um lado, e em vários cursos de ciências sociais (não apenas de Sociologia), por outro, a experiência letiva acabada de descrever mostra que os objetivos/princípios e estratégias pedagógicas discutidas na literatura revista se podem aplicar, com mais ou menos adaptações, no ensino/aprendizagem de MTIS no âmbito de uma licenciatura em Sociologia. Contudo, a experiência letiva anteriormente descrita também contribui para reforçar as principais limitações desta literatura, bem como para mostrar como é que estas podem ser ultrapassadas.

Avaliação da estratégia pedagógica: pontos de vista do docente e dos estudantes

Ao longo dos anos o docente foi realizando, conjuntamente com os estudantes, avaliações informais (discussão em sala de aula) sobre o modo de funcionamento da UC e a estratégia pedagógica adotada. Das notas registadas pelo docente, destacam-se as que são apresentadas seguidamente.

São várias as vantagens identificadas pelo docente. Primeiro, a generalidade dos estudantes exibe um elevado envolvimento/comprometimento com a UC (os níveis de assiduidade e de envolvimento com as atividades são elevados). Segundo, 
os estudantes tomam consciência mais facilmente do que fazem bem e do que fazem menos bem, e desta forma ficam mais motivados para futuras aprendizagens. Terceiro, o stress exibido pelos estudantes no início do semestre diminui após a realização das primeiras aulas de índole mais prática e da primeira atividade do portefólio de grupo e, concomitantemente, assiste-se a um aumento progressivo dos seus níveis de confiança. Quarto, a taxa de aprovação dos estudantes que optam pela modalidade de avaliação contínua é normalmente de $100 \%$.

Por sua vez, os estudantes também têm identificado várias vantagens. Primeiro, afirmam gostar da UC, pois esta inclui muitas atividades práticas. Segundo, sublinham que aprendem mais através da realização de atividades práticas do que através da simples escuta do professor. Terceiro, valorizam bastante os comentários e sugestões realizadas pelo docente sobre as atividades do portefólio de grupo, sublinhando que constituem um contributo essencial para a sua aprendizagem, para além de permitirem melhorar as atividades e, consequentemente, a classificação final. Por último, também referem que a entrega faseada das atividades do portefólio evita que deixem "tudo para a última hora".

A grande desvantagem identificada pelo docente é a significativa quantidade de tempo que é exigida pela avaliação contínua, nomeadamente a leitura das atividades do portefólio de grupo e os comentários sobre as mesmas. O docente reconhece, ainda, que este modelo de funcionamento da UC será mais difícil de implementar em turmas de grande dimensão.

Da parte dos estudantes, a principal desvantagem identificada consiste também no número significativo de horas que têm que dedicar à UC, embora reconheçam que é deveras compensador.

\section{Conclusões}

Conforme foi clarificado na introdução, este artigo tem como objetivo contribuir para a discussão da pedagogia dos MTIS, discussão esta que é premente, particularmente em Portugal. Por outras palavras, este artigo pretende fazer um contributo para o desenvolvimento de uma cultura pedagógica, que, segundo Wagner et al. (2011), inclui promover o debate, a investigação e a avaliação sobre todos os aspetos que se relacionam com o ensino/aprendizagem de MTIS.

Após a reflexão realizada neste artigo, importa delinear algumas notas conclusivas. Primeiro, os princípios e as estratégias pedagógicas usadas pelo docente vão, em termos gerais, ao encontro da literatura revista sobre a pedagogia dos MTIS não só no que respeita ao que esta literatura propõe em termos de objetivos pedagógicos, mas também no que propõe em termos de PCK genérico, PCK 
específico para os MTIS e PCK específico para os métodos qualitativos. Isto mostra que, não obstante as especificidades da literatura revista, é possível aplicar os princípios e as estratégias pedagógicas por esta sugerida no contexto do ensino/aprendizagem de uma UC de métodos qualitativos numa licenciatura de Sociologia.

Segundo, não obstante esta convergência geral entre o preconizado pela literatura revista e os princípios e estratégias pedagógicas utilizadas pelo docente, a prática letiva deste último vai para além do que é recomendado pela referida literatura, colmatando algumas das suas principais limitações. Partindo da adesão ao paradigma da aprendizagem ativa, o docente propõe a adoção de três princípios pedagógicos, em que dois deles possibilitam ultrapassar limitações importantes da literatura sobre a pedagogia dos MTIS, nomeadamente: a avaliação contínua deve ser concebida como um elemento do método de ensino/aprendizagem, e a avaliação deve ser precedida por treino. Estes princípios suportam uma estratégia pedagógica que aposta fortemente na interligação entre aulas teórico-práticas e avaliação contínua, aposta que tem sido avaliada pelo próprio docente e pelos estudantes de uma forma francamente positiva, embora alguns aspetos menos positivos se tenham vindo a identificar.

Terceiro, embora a melhoria da qualidade do ensino/aprendizagem seja uma prioridade do denominado Processo de Bolonha, o desenvolvimento das competências pedagógicas dos professores do ensino superior faz-se, tal como reconhece o Relatório de Implementação do Processo de Bolonha (European Commission/EACEA/Eurydice, 2018), através de uma aprendizagem no trabalho (learning on the job), pois a formação pedagógica não é um requisito para o acesso à profissão de docente neste nível de ensino. Assim, os princípios e as estratégias pedagógicas discutidas na literatura revista, algumas das quais preconizadas pelo Processo de Bolonha como, por exemplo, a aprendizagem ativa, acabam por não estar disseminadas no ensino superior devido à inexistência de uma política coerente de formação pedagógica dos professores deste nível de ensino. Em última instância, isto contribui para a inexistência de uma verdadeira cultura pedagógica no ensino superior.

Quarto, este artigo não tem como objetivo estabelecer um conjunto de padrões de ação ou de boas práticas no que respeita ao ensino/aprendizagem de MTIS, pois tal desígnio poderia limitar o desenvolvimento de uma cultura pedagógica e obscurecer as especificidades subjacentes não só a cada UC, mas também a cada unidade orgânica e mesmo a cada Universidade. O objetivo deste artigo é apenas o de contribuir para a discussão da pedagogia dos MTIS no ensino superior.

Por último, é importante que esta discussão em torno da pedagogia dos MTIS (e da pedagogia no ensino superior em geral), prossiga no futuro, através da 
integração não só das perspetivas dos professores/formadores, mas também das perspetivas dos estudantes/formandos. Esta discussão poderá realizar-se não só no interior de cada Universidade - em workshops criados para o efeito ou em sessões de debate promovidas pelos conselhos pedagógicos - mas também em eventos de âmbito nacional e internacional que promovam a partilha de experiências pedagógicas. Também é importante que esta discussão se desenvolva com base em estudos empíricos e não apenas a partir de reflexões pessoais. Seria útil que estes estudos procurassem identificar e compreender as ações protagonizadas não só pelos professores, mas também pelos estudantes, com o intuito de promover a aquisição de conhecimentos e competências em termos de MTIS.

\section{Notas}

Por decisão pessoal, o autor do texto escreve segundo o novo acordo ortográfico.

\section{Referências}

Alastalo, M. (2008). The History of Social Research Methods. In P. Alasuutari, L. Bickman, \& J. Brannen (Eds.), The Sage Handbook of Social Research Methods (pp. 26-41). Los Angeles: Sage Publications.

Bonwell, C. C., \& Eison, J. A. (1991). Active Learning: Creating Excitement in the Classroom. 1991 ASHE-ERIC Higher Education Reports. Washington, D.C.: School of Education and Human Development, The George Washington University.

Crothers, C., \& Platt, J. (2010). The History and Development of Sociological Social Research Methods. In C. Crothers (Ed.), Historical Developments and Theoretical Approaches in Sociology (Vol. 1, pp. 44-64). Singapore: EOLSS Publishers/UNESCO.

Earley, M. A. (2014). A synthesis of the literature on research methods education. Teaching in Higher Education, 19(3), 242-253.

European Commission/EACEA/Eurydice. (2018). The European Higher Education Area in 2018: Bologna Process Implementation Report. Luxembourg: Publications Office of the European Union.

Howard, C., \& Brady, M. (2015). Teaching social research methods after the critical turn: challenges and benefits of a constructivist pedagogy. International Journal of Social Research Methodology, 18(5), 511-525.

Kilburn, D., Nind, M., \& Wiles, R. (2014). Learning as Researchers and Teachers: The Development of a Pedagogical Culture for Social Science Research Methods? British Journal of Educational Studies, 62(2), 191-207.

Lewthwaite, S., \& Nind, M. (2016). Teaching Research Methods in the Social Sciences: Expert Perspectives on Pedagogy and Practice. British Journal of Educational Studies, 64(4), 413-430. 
Loughran, J., Berry, A., \& Mulhall, P. (2012). Pedagogical Content Knowledge. In J. Loughran, A. Berry, \& P. Mulhall (Eds.), Understanding and Developing Science Teachers' Pedagogical Content Knowledge. (PROFL, Vol. 12, pp. 7-14). Rotterdam: SensePublishers.

Nind, M. (2019). A new application for the concept of pedagogical content knowledge: teaching advanced social science research methods. Oxford Review of Education, 46(2), 185-201. https:// doi.org/10.1080/03054985.2019.1644996.

Nind, M., Kilburn, D., \& Luff, R. (2015). The teaching and learning of social research methods: developments in pedagogical knowledge. International Journal of Social Research Methodology, 18(5), 455-461.

Wagner, C., Garner, M., \& Kawulich, B. (2011). The state of the art of teaching research methods in the social sciences: towards a pedagogical culture. Studies in Higher Education, 36(1), 75-88.

Data de submissão: 10/12/2019 | Data de aceitação: 05/10/2020 